\title{
Senear-Usher syndrome with parathyroid adenoma
}

\author{
RODNEY S.W.BASLER \\ Department of Dermatology, The University of Michigan, Ann Arbor, Michigan 48104, U.S.A.
}

Accepted for publication II January 1974

SUMMARY

A patient with pemphigus erythematosus and parathyroid adenoma is discussed. He improved dramatically after excision of the tumour.

There has been recent interest in the association between bullous erythema multiforme (Basler, I973), bullous pemphigoid (Parsons \& Savin, I968), pemphigus vulgaris (Saikia, 1972) and Senear-Usher syndrome (Saikia \& MacConnell, I972) with various neoplasms. A 46-year-old male patient presented with pemphigus erythematosus which was refractory to various forms of therapy including large doses of prednisone and parenteral methotrexate. In the course of the investigations, a parathyroid adenoma was discovered. After surgical removal of this tumour, the patient's skin disease dramatically improved, suggesting an association between the two entities. Such an association of these two uncommon entities has not been previously reported.

\section{CASE REPORT}

A 46-year-old Spanish-speaking Mexican-American migrant worker presented with a 2-year history of vesicles and bullae over the chest, back, upper arms, and face. The disease had been controlled with low doses of dexamethasone in the range of 5-10 mg/day by his private physician in Mexico. However, the condition had become more severe over the previous 2 months. No other pertinent history could be obtained.

Physical examination revealed a normally developed Mexican-American male with a bullous eruption over the chest (Fig. I), back, and face. Tense, $0.5-2.0 \mathrm{~cm}$ bullae on an erythematous base were noted, with confluence of the lesions over the forehead and cheeks. A few lesions were also found on the scalp, but none was present in the mouth. Bilateral pterygia were present in the eyes. There were no palpable masses in the neck. Examination of chest, heart, abdomen and neurological system was unremarkable.

Skin biopsies stained with Haematoxylin and Eosin showed intraepithelial subcorneal clefts with acantholysis (Fig. 2). A perivascular, inflammatory infiltrate was also present within the upper dermis consistent with pemphigus erythematosus. Direct immunofluorescence staining demonstrated IgG in the intercellular spaces and at the dermo-epidermal junction. Laboratory screening tests showed a mild anaemia with a haemoglobin of I I.2 $\mathrm{g} / \mathrm{IO0} \mathrm{ml}$, increased calcium of $12.3 \mathrm{mg} / \mathrm{IOO} \mathrm{ml}$, increased 


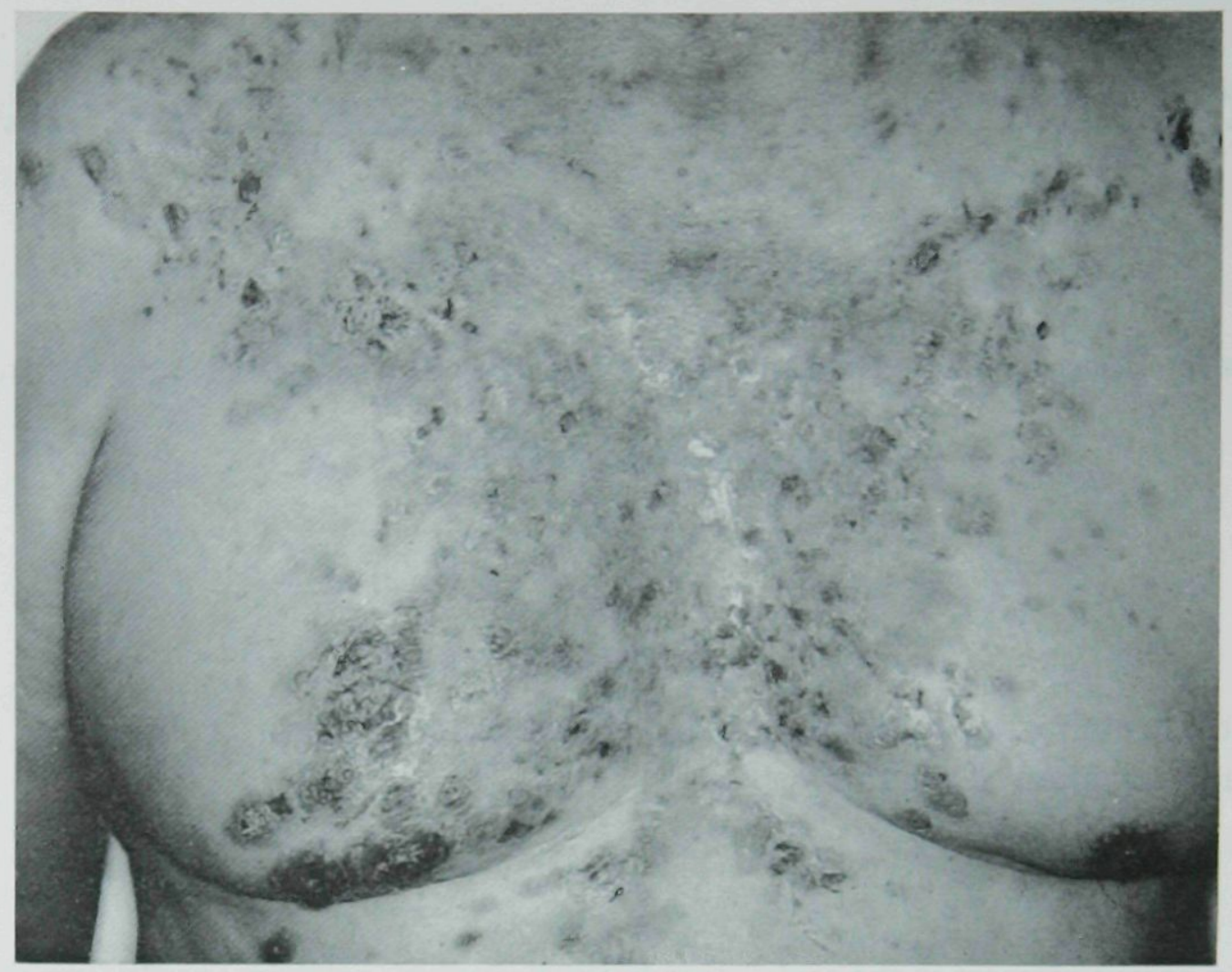

FIGURE I. Vesicular and bullous eruption on erythematous base over chest.

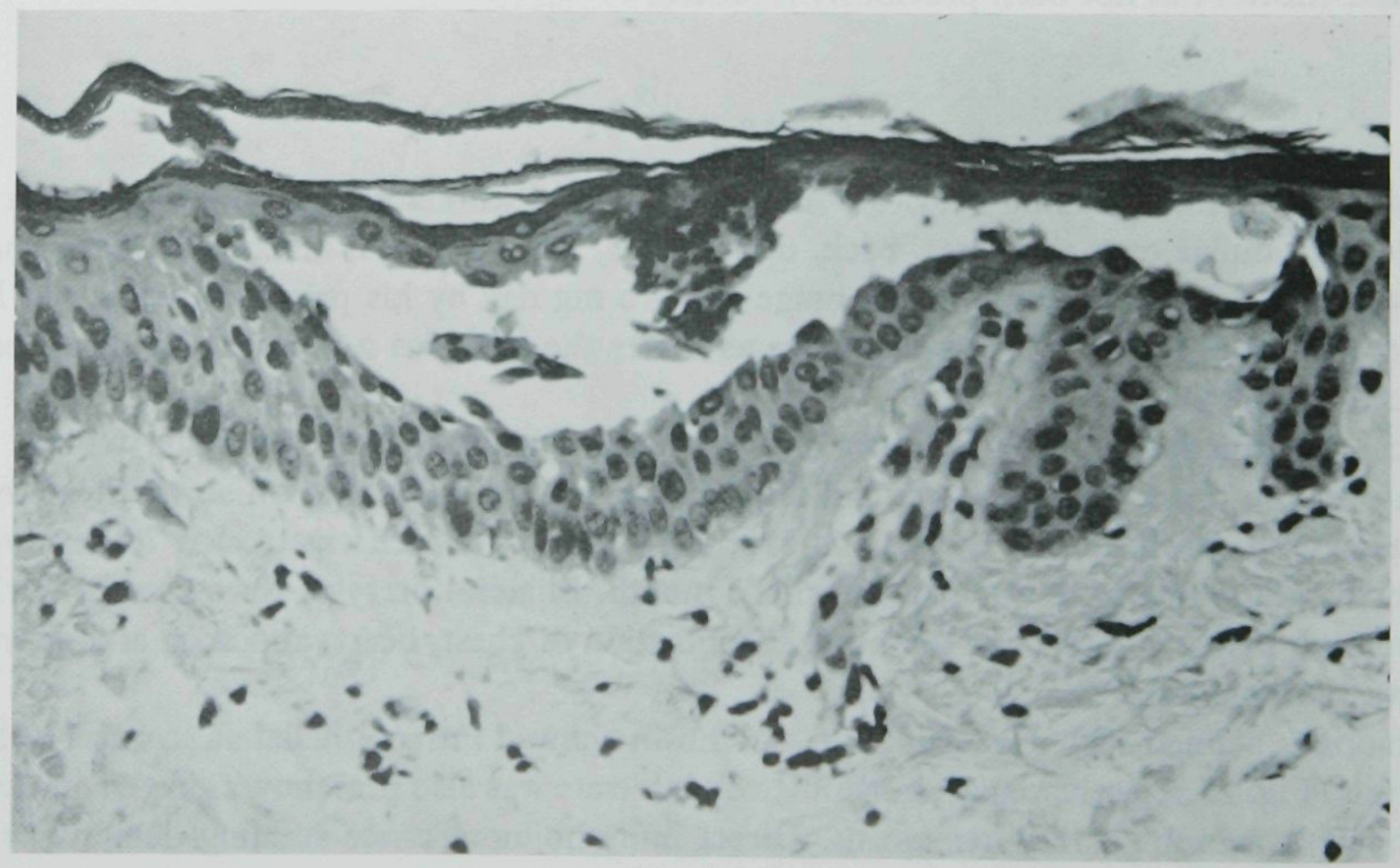

FIGURE 2. Acantholytic, intra-epithelial bulla $(\mathrm{H} \& \mathrm{E}, \times 280)$. 
alkaline phosphatase of $136 \mathrm{iu} / \mathrm{ml}$, and a serum phosphorus at the lower limit of normal at $2.6 \mathrm{mg} / 100$ $\mathrm{ml}$.

The patient was placed on $160 \mathrm{mg}$ of prednisone daily with initial improvement of old lesions, but appearance of a few new vesicles. An attempt to maintain this dosage during the investigation of his hypercalcaemia was unsuccessful; the pemphigus remained active and steroid side effects of melaena and transient psychosis appeared. The patient was started on $25 \mathrm{mg}$ intramuscular methotrexate weekly and tapering of the steroid was begun.

Results of a parathyroid hormone assay showed a significantly increased value of $403 \mathrm{pg} / \mathrm{ml}$, and the patient was transferred to the surgical service. Exploration of the neck revealed gross enlargement of the right inferior parathyroid, which was excised. The pathological report confirmed the diagnosis of a parathyroid adenoma.

The patient improved dramatically in the first $48 \mathrm{~h}$ following the operation, after nearly 6 weeks of unsuccessful therapy with conventional medications. He was discharged on the fifth post-operative day on $20 \mathrm{mg}$ of prednisone daily with no active disease. The dosage of prednisone has gradually discontinued without new lesions appearing. New vesicles appeared approximately 3 months after the operation, however, and the patient was again placed on prednisone. The eruption has been under good control for the last 5 months on $25 \mathrm{mg}$ every other day. There is no clinical or laboratory evidence of renewed parathyroid hyperactivity associated with the return of the skin disorder.

\section{DISCUSSION}

Three patients with Senear-Usher syndrome and malignant disease were recently reported by Saikia (I972). Although immunological investigations were not done, the clinical history of these patients suggested an interrelationship between their tumours and pemphigus antibodies (Saikia, I973). Such an interrelationship had been demonstrated earlier with a malignant lymphoid neoplasm (Saikia, I972). The fact that our patient's bullous disease responded dramatically to removal of the parathyroid adenoma after a prolonged and unsuccessful course of conventional treatment, suggests that antigens produced by the tumour may have aggravated the clinical condition by stimulating increased production of anti-epithelial antibodies. Though the pemphigus erythematosus did return, the course has been characteristically benign, and the process is under control with a low-dose, everyother-day steroid regimen.

\section{REFERENCES}

BASLER, R.S.W. (1973) Bullous erythema multiforme with benign neoplasm. Fournal of the American Medical Association, 225, 995.

Parsons, R.L. \& Savin, J.A. (1968) Pemphigoid and malignancy. British fournal of Cancer, 22, 669.

SAIKIA, N.K. (1972) Extraction of pemphigus antibodies from a lymphoid neoplasm and its possible relationship to pemphigus vulgaris. British fournal of Dermatology, 86, 4I I.

SAIKIA, N.K. (1973) Pemphigus and malignancy. British fournal of Dermatology, 88, 409.

SAIKIA, N.K. \& MACCONNELL, L.E.S. (1972) Senear-Usher syndrome and internal malignancy. British fournal of Dermatology, 87, I. 
This document is a scanned copy of a printed document. No warranty is given about the accuracy of the copy. Users should refer to the original published version of the material. 